\title{
Never mind the quality feel the width or less is more?
}

\author{
Derek Richards \\ Editor, Evidence-based Dentistry
}

Never Mind the Quality Feel the Width was a mid-sixties British sitcom, but it is the title rather than the plot, based on two tailors in business together, that is most relevant here. We are currently being overwhelmed with information. Bastian et al ${ }^{1}$ highlight this in a recent paper where they point out that 75 trials and 11 systematic reviews are published each day and that this has not yet plateaued. Now fortunately not all of these trials and systematic reviews are dentally relevant, but a number of them will be and a number of papers ${ }^{2-4}$ have identified how many journals you need to scan and how many high quality articles you need to read per week to stay current in those specialities, these are summarised in Table 1. As the number of both journals and papers published has increased since these papers were published the number will undoubtedly have increased.

At one level this may seem advantageous to a journal whose aim is to summarise high quality articles that are relevant to dental practitioners. Sadly, however, this increase in the availability and number of trials and systematic reviews does not mean that there has been an across-the-board improvement in the quality of these publications. For example a quick and dirty search of PubMed using just the terms periodontal treatment, and pre-term birth and limited to reviews produces 77 hits with at least seven meta-analyses.

This to me argues a case for focussing on high quality reviews and trials, an approach increasingly being taken by some funding agencies as we move into an era with greater financial pressure on research. Some funding agencies now insist that system-

\begin{tabular}{|l|l|l|l|}
\hline \multicolumn{1}{|l|}{ Table 1. Number of journals and number of high quality articles in $\mathbf{3}$ dental specialities } \\
\hline & Prosthetics & Paediatric Dentistry & Endodontics \\
\hline No of journals & 60 & 75 & 123 \\
\hline No of papers & 8 & 24 & 3
\end{tabular}

atic reviews are done in an area to identify deficiencies before funding. Clarke et $a l^{5}$ in a recent status report on a call for reports of clinical trials to begin and end with upto-date systematic reviews of other relevant evidence showed that there has been no evidence of progress between 1995 and 2007, and while this focussed on medical journals I doubt the situation is any different in dental journals. I am aware that we don't always reference previous relevant systematic reviews even in the commentaries in this journal, although we do try!

In attempting to stem the flow of duplication of effort, a number of clinical trial registries exist that allow researchers to register the protocols of their trials. While The Declaration of Helsinki (www. wma.net/en/30publications/10policies/ b3/index.html) states that 'Every clinical trial must be registered in a publicly accessible database before recruitment of the first subject'. There are a number of free registries that accept entries, and the WHO has search platform (http://www. who.int/ictrp/en/) that allows searching across various databases. This portal should be the first port of call for anyone considering a trial, in order to minimise duplication. Recently a similar portal for registering ongoing systematic reviews called PROSPERO (see news section for further details) has been launched to encour- age prospective registration of systematic review protocols, again with the aim of reducing duplication of effort.

Bastian et al $^{1}$ repeated an earlier call from Chalmers and Glasziou ${ }^{6}$ for effective prioritisation to 'reduce avoidable waste in the production and reporting of research evidence'. They also pointed out that we need a leaner and more efficient ways of staying up to date noting that even the Cochrane Collaboration is having difficulty keeping all it reviews up-to-date. While no-one wants to stifle the search for new knowledge there is a need to focus efforts on high quality studies to reduce the numbers of unnecessary trials and to prioritise areas for systematic reviewing.

1. Bastian $\mathrm{H}$, Glasziou P, Chalmers I. Seventy-five trials and eleven systematic reviews a day: how will we ever keep up? PLoS Med. 2010 7: e1000326.

2. Nishimura K, et al Benchmarking the clinical prosthetic dental literature on MEDLINE. J Prosthet Dent. 2002 88: 533-541.

3. Yang $\mathrm{S}$ et al $\mathrm{A}$ bibliometric analysis of the pediatric dental literature in MEDLINE. Pediatr Dent. 2001 Sep-Oct; 23: 415-418

4. Kim MY et al Benchmarking the endodontic literature on MEDLINE. J Endod. 2001 27: 470-473.

5. Clarke M, Hopewell S, Chalmers I. Clinical trials should begin and end with systematic reviews of relevant evidence: 12 years and waiting. Lancet. 2010 376: 20-21

6. Chalmers I, Glasziou P. Avoidable waste in the production and reporting of research evidence. Lancet. 2009 374: 86-89.

Evidence-Based Dentistry (2011) 12, 2. doi:10.1038/sj.ebd.6400767 